\title{
Assessment Of Jordanian Nurses' Knowledge To Perform Glasgow Coma Scale
}

\author{
Hamza Al-Quraan, MSN, RN, CNS \\ BMT Nurse, King Hussein Cancer Center, Amman, Jordan \\ Mohannad Eid AbuRuz, PhD, RN, CNS \\ Associate Professor, Applied Science Private University, Amman, Jordan \\ doi: 10.19044/esj.2016.v12n27p208 URL:http://dx.doi.org/10.19044/esj.2016.v12n27p208
}

\begin{abstract}
The most important assessment of neurological examination in the clinical setting is assessing level of consciousness. The first neurological tool used to assess patients' level of consciousness was the Glasgow Coma Scale. It is considered as the most common less subjective gold standard coma assessment tool. The purpose of this study was to assess Jordanian nurses' knowledge about Glasgow Coma Scale. A non-experimental, descriptive cross-sectional correlational design was performed in four (3 private hospitals and 1 governmental) hospitals in Amman-Jordan. A self-reported questionnaire was answered by all (ICU, CCU, ER, and Telemetry) nurses who accepted to participate in the study. A total of 200 questionnaires were distributed to the participants with $90 \%$ response rate ending with 180 questionnaires in the final analysis. More than half of the sample $(56.7 \%)$ was males. The participants were young nurses with mean age of $26.3 \pm 8$ years. The total mean score for the whole sample was $7.38 \pm 1.96$. There was no relationship between experience, level of education, and training course and knowledge level. Nurses working in accredited hospitals and governmental hospitals recorded more level of knowledge than other hospitals. Emergency Room nurses recorded less level of knowledge than other area of practice. In conclusion, knowledge about Glasgow Coma Scale is a global problem. Jordanian nurses, as other nurses, have inadequate knowledge to perform Glasgow Coma Scale assessment. It is vital and necessary to include educational programs about Glasgow Coma Scale for nurses in all areas of practice and in the curricula of nursing colleges.
\end{abstract}

Keywords: Glasgow Coma Scale, Neurological Assessment, Knowledge, Performance, Nurses, and Jordan 


\section{Introduction}

Nursing is a dynamic and evolving profession, in which knowledge is central to its accountability (Hall, 2005). Nurses need to a quire a wide range of theoretical and practical knowledge in order to provide the appropriate level of care for patients (Hall, 2005). Nurses are responsible to continuously assess patients. One of the major challenging that nurses find during assessment is the neurological dysfunctions; especially in patients with coma. The most important assessment of neurological examination in the clinical setting is assessing level of consciousness (LoC) which is considered the first step (Jaddoua et al. 2013).

Rapid and correct assessment will minimize the neurological complications, unnecessary and incorrect diagnostic procedures, mortality and morbidity. The basic requirements for any assessment to be effective are the availability of an objective, valid, reliable and accurate tools. The first neurological tool used to assess patients' level of consciousness is the Glasgow Coma Scale (GCS) (Chan \& Matter 2013). It is considered as the most common less subjective coma assessment tool. (Emejulu, 2014).

Even though the GCS is an easy, objective, reliable instrument to assess LoC; it has its own limitations (Thi Hien \& Chae 2011). Over the last decade, some criticism about the GCS started to appear in the literature despite plenty of advantages (Segatore \& Way, 1992). GCS is considered easy to use; however, this character opens it to misinterpretation and misapplication. (Mattar et al., 2014; McLernon, 2014).

Nurses not only need to know how to assess LOC by GCS, but also they need to know how to interpret these numerical values. Previous studies (Mattar et al. 2013) found that nurses who do not use the GCS frequently faced difficulties in its application. Furthermore, they lack the knowledge and the self-confidence in the assessment (Shoqirat 2006, Chan \& Matter 2013). In addition, $92 \%$ of the nurses (Shoqirat, 2006; Chan \& Matter, 2013) reported that it is a complex tool and mentioned motor response assessment as an example of its complexity. Head injuries are usually associated with other injuries such as spinal trauma, which might alter the accuracy of GCS and made it complicated in regard to motor response.

One of the factors that might affect the accuracy of GCS scoring is the knowledge of nurses about how to use/score GCS (Thi Hien \& Chae 2011). Different studies showed that higher levels of knowledge promoted the accuracy when performing GCS scoring (Holdgate 2006, lacono \& Lyons 2001). The first step in such studies should be the assessment of nurses' knowledge in performing GCS. Thereafter, if the results showed that the nurses are lacking the knowledge, then another interventional study would be recommended. 
Thi Hien and Chae (2011) had investigated the accuracy of GCS knowledge and performance among Vietnamese nurses. A cross-sectional descriptive study was conducted using a questionnaire to assess the knowledge and a structured evaluation tool to measure the accuracy of the scores. More than ninety percent of the nurses responded correctly to questions regarding their GCS basic knowledge. However, when it comes to the clinical scenarios requiring the application of the basic knowledge, only $47.9 \%$ of the nurses answered these questions correctly. Moreover, $57.4 \%$ of the nurses answered the questions of the components of the GCS wrong. Based on these results, one can conclude that the nurses were lacking the necessary knowledge about GCS especially when it comes to the clinical setting.

Jaddoua et al (2013) also conducted a study in Iraq to assess the level of nurses' knowledge about the GCS. They also tried to find the relationship between nurses' knowledge and their demographic characteristics including educational level and years of experiences. This study was conducted at three hospitals from $1^{\text {st }}$ of January to the end of April 2011. A purposive sample of 100 nurses answered a 25 -item questionnaire. The results indicated that nurses' knowledge concerning all items related to GCS was inadequate. The recommendation of this study was a critical need to educate the nurses about the GCS.

In an experimental study done by Hansen et al. (1992), they controlled the confounding variables and randomly assigned the participants to two groups. The interventional group attended a lecture about GCS by a specialist nurse. The second group was a control. The results showed that the interventional group has better knowledge than the control group. These results were better in particular for assessing the verbal and motor response. Moreover, this study eliminated the effect of experience by enrolling participants who were first year nurses working in similar setting. However, the limitation of this study was because it was not clear if a single rater or multi raters did the assessment, which question the inter-rater reliability of the study.

Heim, et al. (2009) had checked the knowledge of GCS by air-rescue physicians using prospective anonymous observational study. Among 103 participants, $36.9 \%$ answered the clinical case wrongly. Wrong evaluation of the motor component occurred in 28 questionnaires, and 19 errors were made for the verbal score. Consultants made significantly less errors than the rest of the participating physicians. Less experienced physicians had a higher rate of errors. The results of this study indicated that although the theoretical knowledge of the GCS was correct, significant errors were made in scoring a clinical case. Further emphasis on teaching the GCS is mandatory. 
Therefore, the main purpose of this study was to assess Jordanian nurses' knowledge about GCS. The study also aimed at answering the following research questions:

1. Is there a relationship between years of experiences and knowledge about GCS?

2. Is there a relationship between area of practice and knowledge about GCS?

3. Is there a difference in nurses' knowledge based on hospital type or area of practice?

\section{Methodology}

Design

A non-experimental, descriptive cross-sectional correlational design was used to meet the objectives of the study.

\section{Sampling and study sample}

A convenience sample of nurses who meet the following inclusion criteria were included in the study. a) registered nurse with more than 3 months experiences, b) working in any of the following critical care units (ICU, CCU, ER, Neuroscience ICU, Telemetry and Neuroscience ward). Nurses were excluded from the study if they were: a) mangers, b) head nurses, c) supervisors, and d) educators. The reason behind their exclusion is that they are not participating in patients care. Based on the power analysis a sample size of 125 participants were necessary to achieve $80 \%$ power with an alpha of 0.05, and medium effect size (Chan \& Matter 2013), using a 20item questionnaire. Therefore, 180 participants who were included in the study was acceptable number.

\section{Sites and setting}

The study was conducted at ICU, CCU, ER, Neuroscience ICU, Telemetry and Neuroscience ward from three private and one governmental hospitals in Amman, Jordan.

\section{Data collection instruments}

Data collection instrument consisted of three parts. Part one; was used to collect sociodemographics (age, gender, educational level, working hospital, working area, years of experience in nursing and in specialty, and if the nurse has training about GCS).

Part two; was used to assess nursing knowledge about GCS. This part consisted of 15 questions about the GCS. Questions number 6 and 12 are true/false. All other remaining questions are multiple choices. Only one answer is correct. Therefore, the total score that the participant can get range 
from 0-15. Higher scores indicated higher levels of knowledge. This instrument was used before by different studies (Mattar et al. 2013, Chan \& Matter 2013). The authors developed this instrument as the following. Ten of the fifteen questions were adapted from questionnaire developed by Shoqirat (2006, pp.44-45) and waterhouse (2008, pp. 494-495). Five questions (6, 8, 13,14 , and 15) were added based on a critical review of the literature.

The validity and reliability of this instrument was granted by (Mattar et al. 2013). Three experts in neuroscience with at least ten years of experience examined the instrument for its validity. The experts asked for amendment because they felt that the instrument is not sufficient; content validity index was 0.73 . After the amendment was implemented, the content validity index increased to 0.80 . The stability and internal consistency was tested by test-retest method. Seventeen nurses performed the test twice one week a part. The correlation coefficient between the scores was 0.71 indicting that the instrument has a satisfactory reliability.

\section{Ethical consideration and procedure of data collection}

Institutional Review Board (IRB) approval was obtained from Applied Science Private University ethical and research committee. The Principle Investigator (PI) explained the purpose of the study to the hospital manager and approval has been taken prior to data collection. The PI also explained the purpose of the study to nurses, and distributed the questionnaire. The questionnaire has a cover sheet ensuring that the study was anonymous, and no identifiers were required. If the nurses answered the questionnaire and returned it back, then this was considered as consent to participate in the study. Then forms are coded to know which hospitals the forms were coming from. Only aggregate data was used for publication purposes.

\section{Data analysis}

All data were coded, and analyzed by using the Statistical Package for the Social Sciences version 21 (SPSS 21). Then, data analysis was performed as following: The check of outliers was performed. Outliers were checked by examining the frequency distributions for each question, so that the incorrectly coded data were identified. Descriptive statics (i.e. frequency, percentage, mean, and standard deviation) was used to describe the sample characteristics. T-test, ANOVA, or Chi-square test was used to check if there was any difference among the hospitals in regard to the sample characteristics according to the level of the variable.

The overall objective of the study was checked by comparing the means and SD of this study with previous studies (Chan \& Matter 2013, Mattar et al. 2014) done on the same topic. To test research questions one 
and two, a correlation coefficient will be computed. To test research question number three, ANOVA with post hoc was conducted.

\section{Results}

This study took place at the (ICU, CCU, ER, and Telemetry) of four different (private and governmental) hospitals in Amman, Jordan. A total of 200 questionnaires were distributed to the participants with $90 \%$ response rate ending with 180 questionnaires in the final analysis. There was no significant difference in any of the sociodemographic among the hospitals. More than half of the sample $(56.7 \%)$ was males. The participants were young nurses with mean age of $26.3 \pm 8$ years. Other sociodemographic of the sample are presented in table 1.

Table 1: Sociodemographic characteristics of the sample

\begin{tabular}{|c|c|c|}
\hline \multicolumn{2}{|c|}{ Variable } & \multirow{2}{*}{$\frac{\text { Score }(\mathrm{N}=180)}{102(56.7 \%)}$} \\
\hline & \multirow{3}{*}{$\begin{array}{l}\text { Male } \\
\text { Female }\end{array}$} & \\
\hline Gender & & $78(43.3 \%)$ \\
\hline \multirow{4}{*}{ Hospital Type } & & $26.3 \pm 2.8$ \\
\hline & Private & $120(66.7 \%)$ \\
\hline & Public & $60(33.3 \%$ \\
\hline & ICU & $85(46.7 \%)$ \\
\hline \multirow{3}{*}{ Area of practice } & $\mathrm{CCU}$ & $37(20.6 \%)$ \\
\hline & ER & $45(25.0 \%)$ \\
\hline & Telemetry & $14(14.9 \%)$ \\
\hline \multirow{2}{*}{ Education level } & Bachelor & $178(98.9 \%)$ \\
\hline & Master & $2(1.1 \%)$ \\
\hline \multirow{2}{*}{ Years of experience } & Nursing & $3.6 \pm 6.0$ \\
\hline & Specialty & $2.9 \pm 2.1$ \\
\hline \multirow[t]{2}{*}{ Received training } & Yes & $56(31.1)$ \\
\hline & No & $124(68.9)$ \\
\hline
\end{tabular}

Values in table are number (\%) or mean \pm SD.

\section{Total knowledge level for nurses}

The total mean score for the whole sample was $7.38 \pm 1.96$.

There was no statistical difference in knowledge level between those who had training and those who did not have. Moreover, there were no statistical significant relationship between knowledge level and years of experience neither in nursing nor in the specialty.

\section{Levels of knowledge among the hospitals}

ANOVA with post hoc test was performed to compare the means of total knowledge about GCS among the hospitals. There was a significant difference in total knowledge among the hospitals $\left(\mathrm{F}_{(3,175)}=3.42, \mathrm{p}<.05\right)$. Post hoc analysis showed that private hospital number one was the responsible for the significant main effect. This has lower levels of 
knowledge (6.69 \pm 2.30$)$ about GCS compared to governmental hospital and private hospital 2 table 2 .

Table 2: Post hoc LSD test for differences of total knowledge means among hospitals

\begin{tabular}{ccccc}
\hline Hospital & Compared with & Mean \pm SD & Mean Difference & Sig. \\
\hline Private 1 & Private 3 & $7.44 \pm 2.28$ & -0.75 & 0.06 \\
& Governmental & $7.60 \pm 1.30$ & -0.91 & $0.01^{*}$ \\
& Private 2 & $8.04 \pm 1.65$ & -1.34 & $0.01^{*}$ \\
\hline & *: The mean difference is significant at the 0.05 level.
\end{tabular}

\section{Levels of knowledge among area of practice}

ANOVA with post hoc test was performed to compare the means of total knowledge about GCS among the areas of practice. There was a significant difference in total knowledge among the areas of practice $\left(\mathrm{F}_{(3,175)}\right.$ $=3.04, \mathrm{p}<.05)$. Post hoc analysis showed that ER was the responsible for the significant main effect. ER nurses have lower knowledge levels (6.67 \pm 2.17$)$ than all other areas table 3 .

Table 3: Post hoc LSD test for differences of total knowledge means among areas of practice

\begin{tabular}{ccccc}
\hline Department & Compared with & Mean \pm SD & Mean Difference & Sig. \\
\hline ER & ICU & $7.56 \pm 1.99$ & -0.89 & $0.01^{*}$ \\
& CCU & $7.58 \pm 1.59$ & -0.92 & $0.03^{*}$ \\
& Telemetry & $8.07 \pm 1.33$ & -1.40 & $0.02^{*}$ \\
\hline
\end{tabular}

ER: Emergency Room, ICU: Intensive Care Unit, CCU: Critical Care Unit, *: The mean difference is significant at the 0.05 level.

\section{Discussion}

To the researchers' knowledge, this is the first study designed specifically to check Jordanian nurses' knowledge about GCS. The findings of this study showed the following: a) Jordanian nurses lack the basic knowledge about GCS, b) private hospital number one was responsible about the difference in the knowledge level among the hospitals, c) emergency room nurses were responsible about the difference in knowledge among the areas of practice, d) neither the years of experience in nursing nor in the specialty affected the knowledge level, e) training did not affect the knowledge, and f) educational level also did not affect the knowledge.

The mean of total knowledge of all participants was $7.37 \pm 1.95$. The results of previous studies (Shoqirat 2006, Chan \& Matter 2013) done in the same topic revealed a higher mean $(10.8 \pm 2.2)$. Even though, the authors concluded that the nurses lack the basic knowledge about the GCS and recommended different training and teaching programs to be done. This means that our nurses also lacking the basic knowledge. It is very important to have a high knowledge about the GCS and its application because the scores will affect the decisions and the treatment of the patients.

These results are supported in the literature in different studies. Jaddoua et al (2013) conducted a similar study in Iraq and showed that the 
nurses have inadequate knowledge concerning all items related to GCS. The recommendation of this study was a critical need to educate the nurses about the GCS. Similarly, a study in Vietnam showed that the nurses were lacking the necessary knowledge about GCS especially when it comes to the clinical setting (Thi Hien \& Chae, 2011).

This problem appears to affect not only nurses but also physicians. Previous studies showed that physicians also lack knowledge about GCS and its application. Emejulu et al (2014) conducted a study to assess the level of knowledge of GCS among 139 physicians in South-East Nigeria working at a federal teaching governmental hospital. The results showed that the physicians have a poor knowledge not only in recalling but also in the application of the GCS system. The authors recommended that continuing medical education is strongly advocated. Heim et al (2009) checked the knowledge of GCS among 103 air-rescue physicians. The major significant errors were made in scoring a clinical case. The authors recommended that further emphasis on teaching the GCS is mandatory.

Results showed that there was a statistical difference among nurses' knowledge. Private hospital number two recorded the highest mean and the Private hospital number one was the lowest. A further look at these hospitals explains why these results emerged. Private hospital number two is a private hospital which has an international accreditation requiring continuous educational programs to be done to the nurses. Furthermore, the quality programs assures yearly competency to be done to the nurses and requiring unit specific orientation (i.e. ICU, CCU,...) in addition to the general nursing orientation. When these orientation programs were reviewed, a brief session about the neurological assessment was included.

The governmental hospital also recorded higher levels of knowledge than private hospital number one. This governmental hospital has a very high occupancy rate more than private hospitals. This hospital is one of the two governmental hospitals covering Amman city, which is the largest metropolitan area in Jordan. This allows the nurses to perform GCS more frequently. In addition, this is a teaching hospital were medical and nursing students do their training with their preceptors. Different discussions and grand rounds are done daily with multidisciplinary teams in the hospitals. Taking all these into consideration, no wonder that nurses in the governmental hospital have higher levels of knowledge than private hospital number one.

The result showed that ER nurses had the lowest mean among all other areas. Previous studies (Mattar et al. 2013, Hien \& Chae, 2011) also showed that health care workers working in the ICUs have the highest knowledge. ICU nurses are required to perform the GCS hourly for their patients regardless to the type of the case or how much the case is critical. 
On the other hand, ER nurses are not performing GCS for all their patients, and when necessary they will perform this task quickly and infrequently.

There was no relationship between the years of experience and the total. This was for both experiences in nursing and in the specialty. Previous studies (Heron, 2001) also showed inconsistent results regarding this area. Further research is recommended for this purpose. The number of nurses who hold master degree was very small to make meaningful comparisons regarding levels of education. Future research including larger sample size with different educational level is recommended. The study showed there is no statistically difference between nurses had a training course about GCS and nurses who hadn't. Previous studies showed that interactive training was successful in improving the knowledge (Palmer \& Knight, 2006; Woodward, 2007; Waterhouse, 2009). We don't have any idea about what training these nurses get and what was the content or method of teaching of this training. It is highly recommended to do a randomized trial to check the effect of a formal interactive program on the knowledge level.

\section{Conclusion and recommendation}

It seems that knowledge about GCS scale is a global problem. Jordanian nurses as well have inadequate knowledge to perform GCS. There was no relationship between experience, level of education, and training course and knowledge level. Nurses working in accredited hospitals and governmental hospitals recorded more level of knowledge than other hospitals. Emergency Room nurses recorded less level of knowledge than other area of practice. Education of all nurses regardless to the area of practice is recommended. It is also recommended to check the effect of sociodemographics, training and interactive educational programs on knowledge level.

\section{References:}

Chan, M., and Matter, I. (2013). Investigating nurses' knowledge, attitudes and self confidence patterns to perform the conscious level assessment: A cluster analysis. International Journal of Nursing Practice. 19 (4), 351-359. doi:10.1111/ijn.12077.

Emejulu, J., Nkwerem, S., and Ekweogwu, O (2014), Assessment of physicians' knowledge of Glasgow coma score. Nigerian Journal of Clinical Practice, 17 (6), 729-734.

Hall, A., (2005), Defining nursing knowledge. Nursing Times. 101 (48), 3437.

Hansen, R., Norris, H., and Sceriha, N. (1992). The effectiveness of education on the performance of neurological observation: a collaborative study. Australasian Journal of neuroscience. 5 (1), 1-9. 
Heim, C., Schoettker, P., Gilliard, N., and Spahn, D. R. (2009). Knowledge of Glasgow coma scale by air rescue physicians. Scandinavian Journal of Trauma, Resuscitation and Emergency Medicine. 17 (39), 1-6. doi:10.1186/1757-7241-17-39.

Heron, R. (2001). Inter-rater reliability of the Glasgow coma scale among nurses in sub-specialties of critical care. Australian Critical Care. 14 (3), 100-105.

Holdgate, A., Ching, N., and Angonese, L. (2006). Variability in agreement between physicians and nurses when measuring the Glasgow Coma Scale in the emergency department limits its clinical usefulness. Emergency Medicine Australasia. 18 (4), 379-384.

Jaddoua, B., Mohammed, W., and Abbas, A. (2013). Assessment of Nurse's Knowledge Concerning Glasgow Coma Scale In Neuro Surgical Wards. Journal of Kufa for Nursing Science. 3 (2) 1-10.

Lacono, L., and Lyons, K. (2001). Making GCS as easy as 1 , 2 , 3 , 4 , 5 , 6. Journal of Trauma Nursing. 12 (3), 77-81.

Mattar, I., Liaw, S., and Chan, M. (2013), A study to explore nurses' knowledge in using Glasgow coma scale in acute care hospital. Journal of Neuroscience Nursing 45 (5), 272-280.

Mattar, I., Liaw, S., and Chan, M. (2014). Nurses' self-confidence and attitudes in using the Glasgow Coma Scale: a primary study. British Association of Critical Care Nurses, 20 (2), 98-107. doi: 10.1111/nicc.12077 McLernon, S. (2014). The Glasgow Coma Scale 40 years on: a review o f its practical use. British Journal of Neuroscience Nursing. 10 (4), 179-184.

Palmer, R., and Knight, J. (2006). Assessment of altered conscious level in clinical practice. British Journal of Nursing. 15 (22), 255-259.

Segatore, M., and Way, C. (1992). The Glasgow Coma Scale: Time for change. Heart Lung. 21(6), 548-557.

Shoqirat, N. (2006). Nursing students' understanding of the Glasgow Coma Scale. Nursing Standard. 20 (30), 41-47.

Thi Hien, N., and Chae, S. (2011). The Accuracy of Glasgow Coma Scale Knowledge and Performance among Vietnamese Nurses. Perspectives in Nursing Science. 8 (1), 54-61.

Waterhouse, C. (2008), An audit of nurses'conduct and recording of observations using the Glasgow coma scale. British Journal of Neuroscience Nursing, 4 (10), 492-499.

Waterhouse, C. (2009). The use of painful stimulus in relation to Glasgow Coma Scale observations. British Journal Neuroscience Nursin. 5 (5), 209215.

Woodward, S. (2007). Conference sparks great pain debate. British Journal of Neuroscience Nursing. 3 (4), 137-137. 